\title{
MANUFACTURING METHODS FOR INDIVIDUAL ALIGNERS AND TRAINERS FROM THERMOPLASTS AND CLINICAL INDICATIONS FOR THEIR APPLICATION
}

\section{S.V. Dmitrienko' , D.A. Domenyuk², M.N. Puzdyryova}

\author{
'Department of Dentistry, Pyatigorsk Medical-Pharmaceutical Institute \\ (Branch of Volgograd State Medical University, Ministry of Healthcare, \\ Russian Federation, 11, pr. Kalinina, Pyatigorsk-32, Stavropol Region, \\ Russia 357532. E-mail:s.v.dmitrienko@pmedpharm.ru, \\ tel: +78793324474 \\ ${ }^{2}$ Department of general practice dentistry and child dentistry, Stavropol \\ State Medical University, Ministry of Healthcare of Russian Federation, \\ 310, Mira Street, Stavropol, Russia 355017. E-mail: domenyukda@mail. \\ ru, tel: +7918870 1205 \\ ${ }^{3}$ Department of pediatric dentistry and orthodontics Saint-Petersburg \\ State Pediatric Medical University, Saint-Petersburg, Russia, st. \\ Lithuanian, 2, 194100. E-mail:spb@gpma.ru, tel:+7812 2950646
}

Standard preorthodontic trainers are now widely used in the clinical orthodontics to treat patients with dentofacial anomalies and deformities $[1,2]$. However, their use is limited due to the mismatch between the size of the trainers and the dental arches. The nowadays available literature offers data on the dental arches linear dimensions that are recommended to be used for identifying the orthodontic treatment tactics $[3,4]$. Modern methods of studying dental arches have been shown in view of the face and head measurements $[5,6]$. The need for forecasting the dental arch shape, taking into account the individual parameters of teeth, has been explained $[7,8]$. Dental arch anomalies reveal a variety of etiopathogenetic features $[9,10]$. There have been advanced methods proposed for orthopedic and orthodontic treatment of children and adults, using advanced prosthetic structures and taking into account the increased aesthetic requirements $[11,12]$. However, it has been shown that many designs of prostheses and devices made from acrylic plastics have a negative effect on the oral mucosa and affect the oral cavity microbiota. Given that, there is specific interest to be taken in the use of orthodontic and orthopedic structures made from thermoplastic materials, which served the basis for this study.

To identify indications for the methods of manufacturing aligners and trainers from thermoplastics of various rigidity.

\section{Article history:}

Received 10 February 2019

Received in revised form 19 March 2019 Accepted 22 March 2019

\section{MATERIAL AND METHODS}

26 children were examined and treated in the period of mixed and permanent occlusion (11-16 years old) with various types of occlusion anomalies caused by disturbed shape and size of the jaws or their abnormal position. While studying the jaws cast models, modern methods of diagnostics were used, also taking into account the classification of the dental arches shape and size. To simulate the shape of the forecasted dental arch, a method of mathematical and graphic reproduction was applied, taking into account the recommended torque and angulation of permanent teeth. Constructive occlusion was identified taking into account the X-ray analysis of the temporomandibular joint elements. The recommendation was to use aligners for 8-10 hours a day while treating patients with impaired shape and size of the dental arches, and to use aligners at night for children with occlusion that is abnormal due to improper jaw location.

Results and discussion. We produced 28 aligners and trainers for treating patients with abnormal dental arches shape and size, and 31 trainers to treat occlusion disturbances caused by the mandible posterior position. While making the aligners, the stamping method (using a Ministar machine, Germany, Scheu-Dent) was employed. The patients had their respective pick-up impressions taken and then cast on two pairs of die stone, one of which was diagnostic, the other being working. The working model was cut into fragments depending on the occlusion anomaly, and preformed in view of the forecasted dental arch shape, which was built through mathematical and graphical modeling. Photocomposite materials were used to eliminate undercuts and close the seams connecting the model fragments on the preformed model. The stamping was performed in the Ministar machine following the manufacturer's recommendations for working with Bioplast materials of various thickness and rigidity. The aligners were made for one or both jaws, depending on the type of occlusal relationships pathology. To treat occlusion anomalies in the sagittal direction, in particular, in case of distal occlusion issues, both aligners were interconnected in a constructive bite. 
Different thickness and rigidity of the aligner ensured due adjustment through treating patients with various types of disclusion. In case of teeth crowding, the aligners proved to be better made of soft and thin material. At the final stage of distal occlusion treatment, after improving the dental arches shape, trainers from hard materials were used. The study outcomes showed that subjective adjustment to the aligners took place in the first week of using the aligner or trainer. The dental arch shape improved during the first year of treatment. Improving the jaws position and the achieving stable occlusive relationship took at least 16 months, which is consistent with the data reported by other experts.

\section{CONCLUSION}

To improve the shape and size of the dental arches, we recommend using tailor-made aligners made of soft and thin thermoplastic material. At the final stage of treating distal occlusion, once the dental arches shape becomes normal, using trainers made from rigid thermoplastic materials can be recommended.

\section{REFERENCES}

1. Korobkeev A.A., Domenyuk D.A., ShKarin V.V., DMitrienKo S.V., WEISHEIM L.D., KoNNOV V.V. Anatomical features of the interdependence of the basic parameters of the dental arches of the upper and lower jaws of man. Medical news of North Caucasus. 2018. - Vol. 13. - № 1-1. - P. 66-69. (In Russ., English abstract). DOI - https://doi.org/10.14300/ mnnc.2018.13019

2. ShKarin V.V., DAVYdov B.N., Domenyuk D.A., DMITRIENKo S.V. Non-removable arch orthodontic appliances for treating children with congenital maxillofacial pathologies - efficiency evaluation. Archiv EuroMedica, 2018; Vol. 8; 1: 97-98.

3. Borodina V.A., Domenyuk D.A., Veisgeim L.D., DMITRIENKo S.V. Biometry of permanent occlusion dental arches - comparison algorithm for real and design indicators. Archiv EuroMedica, 2018; Vol. 8; 1 : 25-26.

4. Domenyuk D., Porfyriadis M., Dmitrienko S. Major telerenthengogram indicators in people with various growth types of facial area. Archiv EuroMedica, 2018; Vol. 8; 1: 19-24.

5. Shkarin V., Domenyuk D., Lepilin A., Fomin I., DMitrienko S. Odontometric indices fluctuation in people with physiological occlusion. Archiv EuroMedica, 2018; Vol. 8; 1: 12-18.

6. Korobkeev A.A., Domenyuk D.A., SHKARIN V.V., DMitrienko S.V. Types of facial heart depth in physiological occlusion. Medical news of North Caucasus. 2018. - Vol. 13. - № 4. - P. 627-630. (In Russ., English abstract). DOI - https://doi.org/10.14300/ mnnc.2018.13122

7. Dmitrienko S.V., Domenyuk D.A. Dentoalveolar specifics in children with cleft palate during primary occlusion period. Archiv EuroMedica, 2018; Vol. 8; 1: 33-34.

8. DomenYUK D.A., LePILIN A.V., Fomin I.V., DMITRIENKo S.V. Improving odontometric diagnostics at jaw stone model examination. Archiv EuroMedica, 2018; Vol. 8; 1: 34-35.

9. Porfiriadis M.P., DMitrienko S.V., Domenyuk D.A., BudAichiev G.M.A. Mathematic simulation for upper dental arch in primary teeth occlusion. Archiv EuroMedica, 2018; Vol. 8; 1: 36-37.

10. LePILIN A.V., Fomin I.V., DomenYuk D.A., DMitrienko S.V. Diagnostic value of cephalometric parameters at graphic reproduction of tooth dental arches in primary teeth occlusion. Archiv EuroMedica, 2018; Vol. 8; 1: 37-38.

11. Dmitrienko S.V., Davydov B.N., V.V. ShKarin, Domenyuk D.A. Algorithm for determining the size of artificial teeth by the morphometric parameters of the face in people with full adentia. Dentistry. 2018; 97(6): 57-60.

12. Domenyuk D.A., Zelensky V.A., DMitrienko S.V., Anfinogenova O.I., Pushkin S.V. Peculiarities of phosphorine calcium exchange in the pathogenesis of dental caries in children with diabetes of the first type. Entomology and Applied Science Letters. 2018; 5(4): 49-64. 